\title{
Boron Neutron Capture Therapy: A New Generation of Targeted Charged-Particle Radiotherapy
}

\author{
Yi-Wei Chen*1, Fong-In Chou², Wen-Sheng Huang ${ }^{3}$, Ko-Han Lin ${ }^{3}$, Po-Shen Pan ${ }^{4}$, Yu-Cheng Kuo ${ }^{5}$, Shih-Ming Hsu' ${ }^{6}$ Jen- \\ Kun Chen ${ }^{7}$, Chun-Wei Wang ${ }^{8}$, Kuan-Hsuan Chen ${ }^{9}$ Tien-Li Lan ${ }^{1}$, Jia-Cheng Lee ${ }^{1}$
}

${ }^{1}$ Department of Oncology, Taipei Veterans General Hospital, Taipei, Taiwan

${ }^{2}$ Nuclear Science \& Technology Development Department, National Tsing-Hua University, Hsinchu, Taiwan

${ }^{3}$ Department of Nuclear Medicine, Taipei Veterans General Hospital, Taipei, Taiwan

${ }^{4}$ Department of Chemistry, Tamkang University, New Taipei City, Taiwan

${ }^{5}$ Department of Radiotherapy, China Medical University Hospital, Taichung, Taiwan

${ }^{6}$ Department of Biomedical Imaging and Radiological Sciences, National Yang-Ming University, Taipei, Taiwan

${ }^{7}$ Institute of Biomedical Engineering and Nanomedicine, National Health Research Institutes, Miaoli, Taiwan

${ }^{8}$ Department of Oncology, National Taiwan University Hospital, Taipei, Taiwan

${ }^{9}$ Pharmacy Department, Taipei Veterans General Hospital, Taipei, Taiwan

Received: 制: January 04, 2018; Published: 制: January 10, 2019

*Corresponding author: Yi-Wei Chen, MD, PhD. Department of Oncology, Taipei Veterans General Hospital, No. 201, Sec. 2, Shi-Pai Rd., Taipei, Taiwan

\begin{abstract}
Radiation therapy plays an important role in standard cancer treatment. However, patients who are resistant to traditional radiation therapy or who have relapsed after conventional radiation therapy are often encountered in clinical practice. There is therefore a need for a new radiation therapy for this type of patient. Although boron neutron capture therapy is not a new concept of radiation therapy, due to technological breakthroughs and conceptual improvements at the start of the $21^{\text {st }}$ century, this therapy, which covers multidisciplinary technologies, such as medical physics, atomic science and technology, boron-containing drug synthesis, radiobiology, and clinical oncology has advanced greatly, and has gradually matured to a clinically useful therapy for patients with cancer. This article provides a brief introduction to the latest breakthroughs and progress in this technology.
\end{abstract}

Keywords: Boron Neutron Capture Therapy; Radiotherapy; Glioblastoma; Theranostic

\section{Introduction}

Boron neutron capture therapy (BNCT) is not a new concept in the field of radiation oncology. In the 1930s, not long after the British physicist James Chadwick (1891-1974, 1935 Nobel Prize winner of physics) proposed verification of the existence of neutrons, some researchers proposed that, after using thermal neutrons to irradiate boron-10 atom (in the natural world, four-fifths of boron is boron 11 , while one-fifth is boron-10; boron-10 is nonradioactive isotope), boron-10 will be cleaved into two particles with high biological effects, namely an $\alpha$ particle (helium nucleus) and a lithium nucleus. These two biologically active particles not only have an excellent damaging effect on tumor tissue and DNA, but also have a range of effects, limited to 5-10 $\mu$ m (a normal cell diameter is about $20 \mu \mathrm{m}$ ), and thus has little damaging effect on the surrounding normal tissues. Therefore, if a specific boron-10- containing drug can be administered selectively to the tumor cells, and a sufficient flux of thermal neutron radiation is applied, it can induce tumor destruction. Therefore, this concept has always been an ideal for targeted charged-particle radiation therapy in the field of clinical radiation oncology [1].

Although the principles seem to be ideal, the implementation of this concept in clinical practice still has many limitations and difficulties. The main technical limitation is whether boroncontaining drugs can be specifically absorbed by tumors. The other is the high-throughput, quality, thermal neutron source. The source of the tumor dose involves a combination of a reactive boron-10 dose, the neutron dose, and the gamma ray dose. In the 1950s, at the Massachusetts General Hospital in the United States, neurosurgeon Dr. William Sweet led the world's first BNCT clinical trial in human 
patients with malignant brain tumors (glioblastoma; WHO grade IV), using the Brookhaven Medical Research Reactor (BMRR) was conducted in Long Island, New York. Although the nuclear technology was not accurate at the time, and the boron-containing drugs used did not have high specificity (Borax; Na2B407·10H2O), this trial was not expected to achieve a particularly good efficacy on malignant brain tumors [2]. However, it was globally considered an important treatment. Japan, Finland, Sweden, the Netherlands, the Czech Republic, Taiwan and other countries worldwide followed the example of this trial and successively proposed various clinical trials using BNCT to treat malignant brain tumors, and also presented the therapeutic advantage of this therapy, of gradually improving the condition of such tumors. In the clinical treatment of brain tumors in Japan, in particular, it was reported that the patient's disease control period post-BNCT was significantly superior to that after traditional photon therapy alone, with patients not only surviving for more than 2 years, but also having excellent quality of life. In addition, for recurrent head and neck cancer, melanoma, liver cancer, and mesothelioma, etc., all countries have reported inspiring clinical trial results [3-8]. Therefore, with further improvement, this highly bioactive targeted radiotherapy will be able to assist a variety of clinical patients.

\section{Development of New Boron-10-Containing Drugs and Breakthroughs in the Concept of Radiobiology}

One of the key technologies for BNCT is the high tumor-specific uptake of boron-10-containing drugs. The main drugs used in the past involved delivery of BPA (L-BPA [L-(4-10borophenyl) alanine]) and BSH (sodium borocaptate). BPA mainly induces a large amount of drug intake by tumor cells due to its structural similarity to the human essential amino acid phenylalanine, while BSH is an icosahedron with a considerable number of boron-10 structures, which can effectively produce therapeutic benefits. In recent years, the international BNCT medicinal chemistry research and development community has attempted to develop various new promising boron-containing drugs, such as GB-10, boric acid etc. [9-11]. If these new boron-containing drugs can be delivered into tumor cells specifically and efficiently, this would form an important basis for the successful clinical treatment implementing BNCT. In addition, the biological benefits of BNCT differ from traditional photon and proton therapies, etc. In addition to tumor tissues, different normal tissues also have different degrees of biological responses to BNCT. Therefore, BNCT introduces the biological concept of the compound biological effectiveness factor. Experimental calculations in the biological laboratory can facilitate an accurate grasp of BNCT dose calculation. In recent years, the concept of $\mathrm{ABE}$ factor also emerged, which can provide a more accurate concept of the BNCT dosage calculation [12].

\section{PET Exam with 18F-Labeled BPA}

As mentioned above, the key to the success of BNCT is specific absorption of drugs containing boron-10. Therefore, it is important to be able to assess the distribution of drugs in the human body effectively. In recent years, a major breakthrough in the BNCT drug research has been the use of nuclear medicine technology to label therapeutic BPA drugs radioactively with fluorine-18. After injection, the patient undergoes positron-emission tomography (PET) to evaluate whether the BPA drug in the human body is specifically distributed in the tumor tissue [13]. This mode of using drugs to assist both treatment and diagnosis is a common concept in nuclear medicine technology: "Theranostic" (a combination of the concepts of "Therapy" and "Diagnostic"). After the patient has completed FBPA-PET, the clinician must assist in interpreting the Tumor/Normal tissue ratio ( $\mathrm{T} / \mathrm{N}$ ratio) of the boron-containing drug (BPA) in the tumors and in normal tissues. It is considered that, only when this $\mathrm{T} / \mathrm{N}$ ratio exceeds 2.5 , will the clinical treatment response be advantageous, and a too-low ratio indicates that the treatment is not suitable [14-15].

\section{Breakthroughs in Neutron Sources}

In recent years, due to the dramatic breakthroughs in atomic science and technology, through the reconstruction of traditional reactors, the neutron source required by BNCT could be upgraded from thermal neutrons to epithermal neutrons, thereby improving tissue penetration depth to treat deeply located tumors. However, most of the international clinical trial institutions are still limited by the need for an atomic reactor to provide neutron sources. In recent years, the international BNCT community has been actively pursuing the development of neutron accelerators, produced by heavy industry technology, to replace traditional atomic reactors as a stable source of neutrons. Compared with the atomic reactor, the accelerator has better controllability and safety, without the problem of nuclear waste disposal, and it is more suitable to serve as a formal medical device in a medical institution. Internationally, Japan, the United States, Italy, Israel, Russia, and other countries all have BNCT programs for the development of accelerator systems [16]. In particular, Japan's accelerator for BNCT is about to complete clinical trials (in malignant brain tumors and recurrent head and neck cancer). It will become one of the regular formal medical devices in the near future. With the help of high-quality accelerator neutron equipment, BNCT can be quickly and routinely promoted in hospitals [17].

\section{Conclusion}

Cancer treatment is an important and global human health issue and breakthroughs in effective treatment are urgently required. By the joint efforts of various experts, the prospects of BNCT implementation has gradually been revealed. In Asia, Japan is the pioneer of BNCT and is about to promote BNCT as a formal standard of care for cancer. In Taiwan, more than 60 patients have been treated with this therapy in clinical trials and as emergency medical treatment with compassionate use. We hope that in the near future, we can help this high-quality treatment mode, with high tumor damage and low peripheral tissue damage, to become the official standard of care for cancer, to allow more patients to obtain more effective tumor control. 


\section{References}

1. Locher GL (1936) Biological effects and therapeutical possibilities of neutrons. Am J Roentgenol Radium Ther 36: 1-13.

2. Sweet WH (1951) The uses of nuclear disintegration in the diagnosis and treatment of brain tumor. N Engl J Med 245(23): 875-878.

3. Barth RF, Vicente MG, Harling OK, Kiger WS, Riley KJ, et al. (2012) Current status of boron neutron capture therapy of high-grade gliomas and recurrent head and neck cancer. Radiat Oncol 7: 146.

4. Suzuki M, Kato I, Aihara T, Hiratsuka J, Yoshimura K, et al. (2014) Boron neutron capture therapy outcomes for advanced or recurrent head and neck cancer. J Radiat Res 55(1): 146-153.

5. Wolfgang Sauerwein, Andrea Wittig, Raymond Moss, Yoshinobu Nakagawa (2012) Neutron Capture Therapy (2012). Springer 41-54.

6. Kankaanranta L, Seppälä T, Koivunoro H, Välimäki P, Beule A, et al. (2011) L-boronophenylalanine-mediated boron neutron capture therapy for malignant glioma progressing after external beam radiation therapy: A Phase I study. Int J Radiat Oncol Biol Phys 80(2): 369-376.

7. Kawabata S, Miyatake S, Nonoguchi N, Hiramatsu R, Iida K, et al. (2009) Survival benefit from boron neutron capture therapy for the newly diagnosed glioblastoma patients. Appl Radiat Isot 67(7-8): 15-18.

8. Lan TL, Chou FI, Huang WS, Lin KH, Lee YY, et al. (2018) Overt tumor regression after salvage boron neutron capture therapy (BNCT) for a recurrent glioblastoma patient. Ther Radiol Oncol 2: 48.

9. Trivillin VA, Garabalino MA, Colombo LL, González SJ, Farías RO, et al (2014) Biodistribution of the boron carriers boronophenylalanine (BPA) and/or decahydrodecaborate (GB-10) for Boron Neutron Capture Therapy (BNCT) in an experimental model of lung metastases. Appl Radiat Isot 88: 94-98.

\section{ISSN: 2574-1241}

DOI: $10.26717 /$ BJSTR.2019.13.002337

Yi Wei Chen. Biomed J Sci \& Tech Res

This work is licensed under Creative

Commons Attribution 4.0 License

Submission Link: https://biomedres.us/submit-manuscript.php
10. Bai YC, Hsia YC, Lin YT, Chen KH, Chou FI, et al. (2017) Effect of Tumor Microenvironment on Selective Uptake of Boric Acid in HepG2 Human Hepatoma Cells. Anticancer Res 37(11): 6347-6353.

11. Chen YW, Liao PC, Zhang YX, Yeh SY, Wu YH, et al. (2018) Synthesis of multiple boron-containing analogs via Ugi-4CR. Res Chem Intermed p. $1-16$.

12. Ono K, Tanaka H, Tamari Y, Watanabe T, Suzuki M, et al. (2018) Proposal for determining absolute biological effectiveness of boron neutron capture therapy-the effect of $10 \mathrm{~B}(\mathrm{n}, \alpha) 7 \mathrm{Li}$ dose can be predicted from the nucleocytoplasmic ratio or the cell size. J Radiat Res.

13. Wang HE, Liao AH, Deng WP, Chang PF, Chen JC, et al. (2004) Evaluation of 4-borono-2-18F-fluoro-L-phenylalanine-fructose as a probe for boron neutron capture therapy in a glioma-bearing rat model. J Nucl Med 45(2): 302-308.

14. Hanaoka K, Watabe T, Naka S, Kanai Y, Ikeda H, et al. (2014) FBPA PET in boron neutron capture therapy for cancer: prediction of (10)B concentration in the tumor and normal tissue in a rat xenograft model. EJNMMI Res 4(1): 70.

15. Watabe T, Hanaoka K, Naka S, Kanai Y, Ikeda H, et al. (2017) Practical calculation method to estimate the absolute boron concentration in tissues using 18F-FBPA PET. Ann Nucl Med 31(6): 481-485.

16. Altieri S, Prott N (2018) A brief review on reactor-based neutron sources for boron neutron capture therapy. Ther Radiol Oncol 2: 47.

17. Suzuki M, Tanaka H, Sakurai Y, Kashino G, Yong L, et al. (2009) Impact of accelerator-based boron neutron capture therapy (AB-BNCT) on the treatment of multiple liver tumors and malignant pleural mesothelioma. Radiother Oncol 92(1): 89-95.

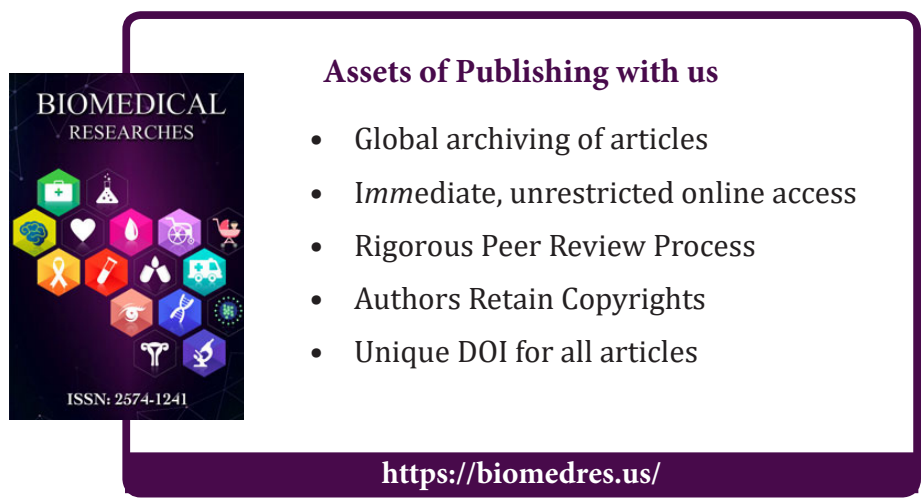

Cite this article: Yi Wei C, Fong In C, Wen Sheng H, Ko Han L, Po Shen P, Yu Cheng K, et al. Boron Neutron Capture Therapy: A New Generation 